\title{
Cryo-Electron Tomography of Microtubules and Granules in Mouse Platelets
}

Juleen Dickson ${ }^{1}$, Wenchun Chen ${ }^{2}$, Joshua Strauss ${ }^{3}$, Renhao Li $^{2}$ and Elizabeth Wright ${ }^{4}$

${ }^{1}$ University of Wisconsin Madison, Madison, Wisconsin, United States, ${ }^{2}$ Emory University School of Medicine, Atlanta, Georgia, United States, ${ }^{3}$ University of North Carolina at Chapel Hill, Chapel Hill, North Carolina, United States, ${ }^{4}$ Department of Biochemistry, University of Wisconsin-Madison, Madison, Wisconsin, United States

Platelets are a major component of the blood and are important for maintaining hemostasis. They are usually found in a resting state, during which they have a discoid shape[1], so they can easily travel through the vessels of the circulatory system. After an injury to a blood vessel, platelets may be exposed to other materials such as the collagen. Once this happens, receptors on the platelets can bind to ligands such as von Willebrand factor, which not only serve to anchor the platelets to the site if the injury, but also contribute to platelet activation.

In order for platelets to become activated, there needs to be a change in the cell morphology. This change in cell morphology involves two major events, 1) Changes in the structure of the cytoskeleton and 2) the release of granules. The changes in the structure of the cytoskeleton are important for the emergence of morphological structures such as filopodia and lamellipodia that are necessary for the platelet adherence and aggregation. Alpha granules contain additional clotting factors such as additional von Willebrand Factor, fibrinogen, factor V, Factor VIII. The dense granules store calcium and ATP which are also important for clotting [2, 3]. Granules perform other functions, for example, during granule release the granule membranes fuse with the platelet plasma membranes, which increases the surface area of the platelets and is important for morphological changes[4]. Thus, understanding the ultrastructure of the resting platelet is important for gaining greater insight into both hemostasis and thrombotic events.

In this study, we used cryo-electron tomography (cryo-ET) to investigate changes to the cytoskeleton, in particular microtubules, in both inactive and activated platelets. The spatial arrangement of microtubules was quantified in the platelet cell body for resting and activated platelets. In addition, we also quantified the spatial orientation of the microtubules in cell extensions, such as filopodia, in platelets in the early stages of activation. The number and surface area of the granules was quantified in resting platelets and those in the early stages of activation. Fresh murine platelets were isolated from blood and were used immediately to prepare cryo-grids with a ThermoScientific Vitrobot plunging system. Cryo-grids were imaged with a Titan Krios $300 \mathrm{kV}$ cryo-TEM equipped with an energy filter and $\mathrm{K} 3$ electron detector. Tilt-series were acquired with SerialEM[5]. Tomographic reconstructions were generated using the IMOD software platform [6] and segmented in either EMAN2[7] or 3dmod application in IMOD[6]. 

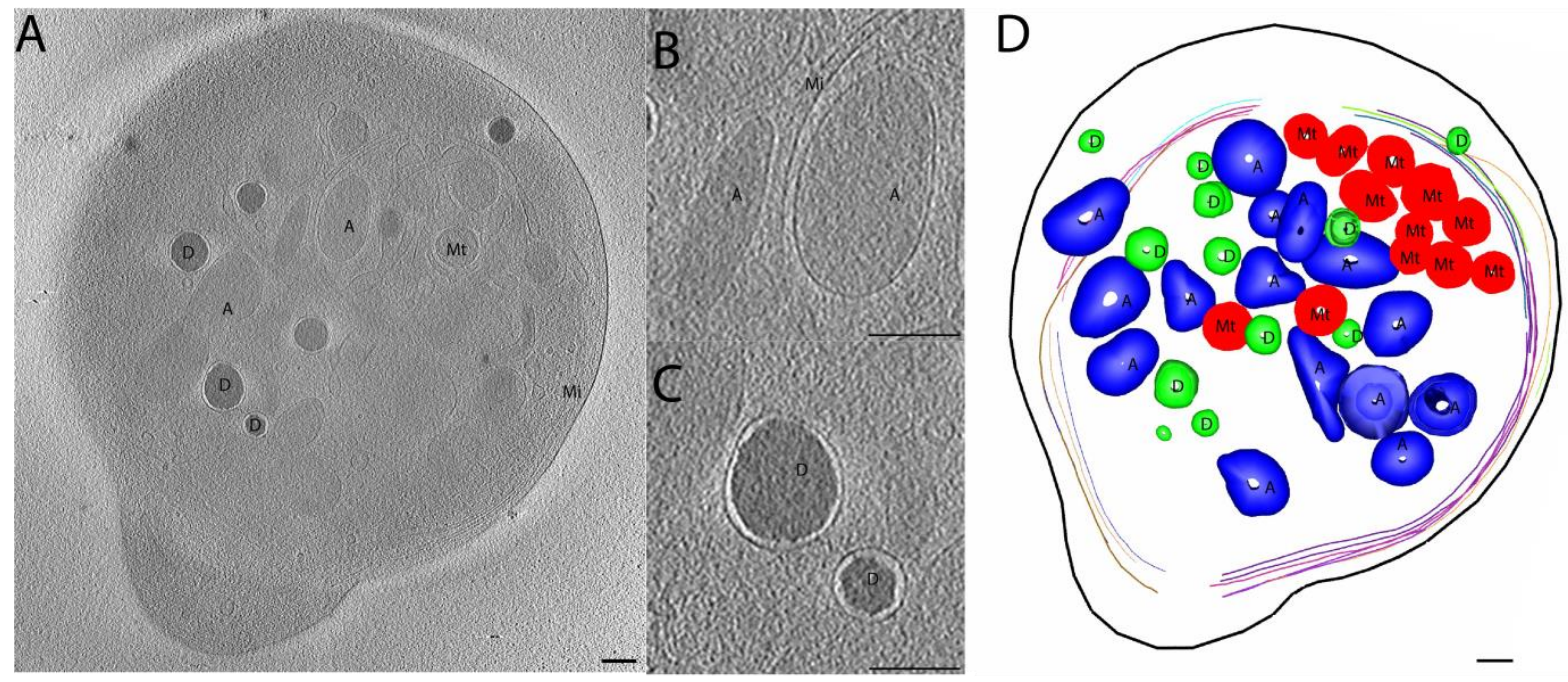

Figure 1. Figure 1. Cryo-electron tomography of a resting mouse platelet. A) A slice through a tomogram of an intact resting platelet. B) Magnified view of an alpha granule (from panel A) next to the microtubules in the marginal band. C) Dense granules next to the microtubules in the marginal band. D) Segmentation of organelles and the microtubule cytoskeleton. $\mathrm{D}=$ dense granules (green), $\mathrm{A}=$ alpha granules (blue), $\mathrm{Mi}=$ microtubules, $\mathrm{Mt}=$ mitochondria (red). Scale bars are $200 \mathrm{~nm}$.
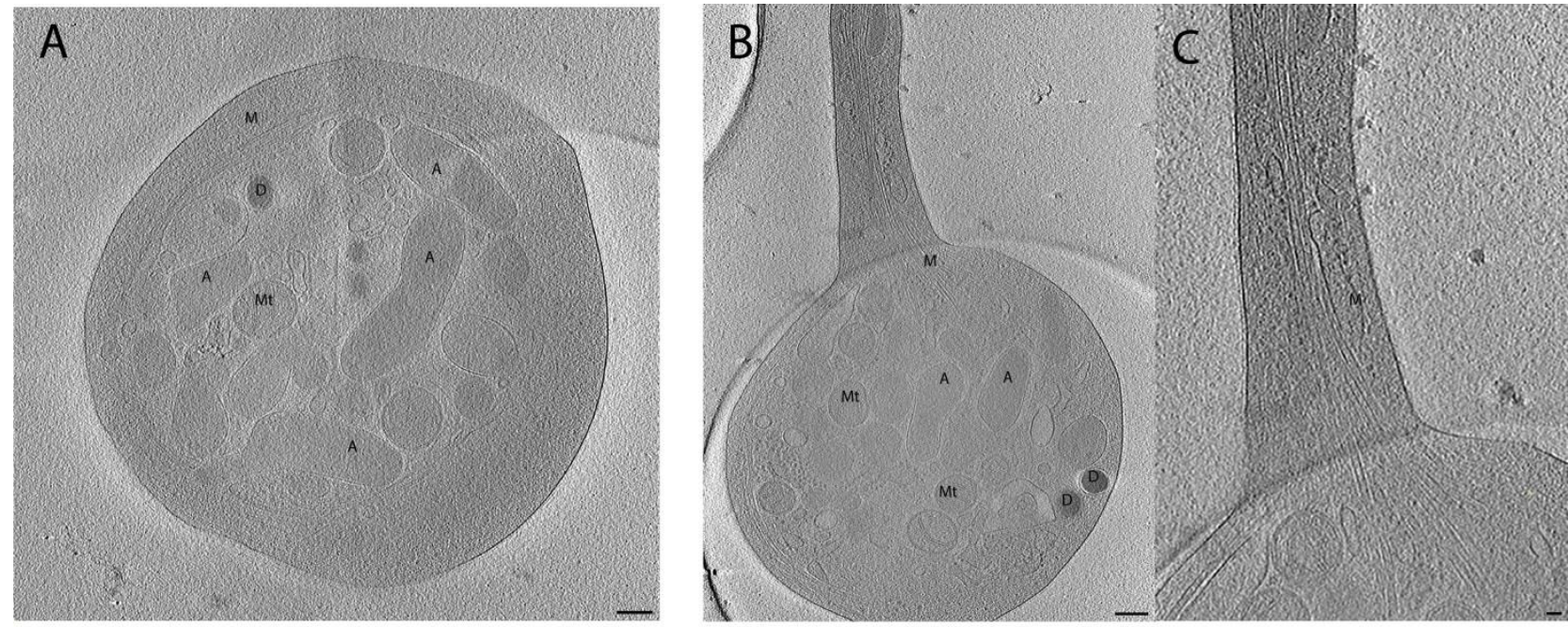

D

Resting
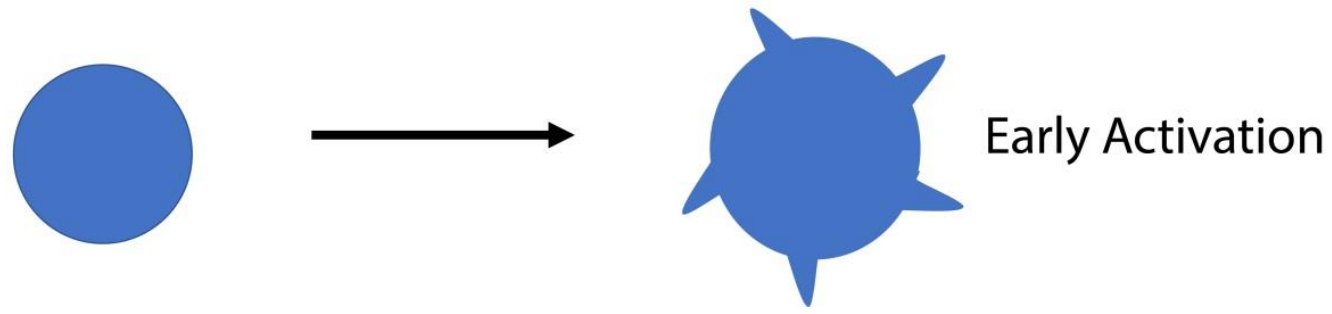

Figure 2. Figure 2. Cryo-electron tomography of a resting and activated mouse platelets. A) A slice through a tomogram of an intact resting platelet. B) A slice through a tomogram of an active platelet with 
filopodia. C) Magnified view of platelet filopodia with extended microtubules (from panel B). D) Diagram showing the morphological changes, such as the emergence of filopodia, that occur during the transition from resting to early activation in platelets. $\mathrm{D}=$ dense granules, $\mathrm{A}=$ alpha granules, $\mathrm{Mi}=$ microtubules, $\mathrm{Mt}=$ mitochondria. (A, B) Scale bars are $200 \mathrm{~nm}$. (C) Scale bar is $50 \mathrm{~nm}$.

\section{References}

1. Shin, E.-K., et al., Platelet Shape Changes and Cytoskeleton Dynamics as Novel Therapeutic Targets for Anti-Thrombotic Drugs. Biomolecules \& therapeutics, 2017. 25(3): p. 223-230.

2. Blair, P. and R. Flaumenhaft, Platelet alpha-granules: basic biology and clinical correlates. Blood Rev, 2009. 23(4): p. 177-89.

3. Hartwig, J.H. and M. DeSisto, The cytoskeleton of the resting human blood platelet: structure of the membrane skeleton and its attachment to actin filaments. J Cell Biol, 1991. 112(3): p. 407-25.

4. $\quad$ Woronowicz, K., et al., The Platelet Actin Cytoskeleton Associates with SNAREs and Participates in $\alpha$-Granule Secretion. Biochemistry, 2010. 49(21): p. 4533-4542.

5. Mastronarde, D., Automated electron microscope tomography using robust prediction of specimen movements. Journal of structural biology, 2005. 152: p. 36-51.

6. Kremer, J.R., D.N. Mastronarde, and J.R. McIntosh, Computer visualization of three-dimensional image data using IMOD. J Struct Biol, 1996. 116(1): p. 71-6.

7. $\quad$ Tang, G., et al., EMAN2: an extensible image processing suite for electron microscopy. J Struct Biol, 2007. 157(1): p. 38-46. 